\section{Extreme Points of Riccati Inequalities}

\section{ANDERS LINDQUIST, C. MARTIN, AND GIORGIO PICCI}

\begin{abstract}
Relations between solutions of the algebraic Riccati equation and the associated quadratic matrix inequalities are discussed and explained.
\end{abstract}

The purpose of this note is to clarify some of the relations that exist between solutions of the algebraic Riccati equation and the associated quadratic matrix inequalities. In particular, the main result of the note is to establish that there are extreme points in the solutions set of quadratic matrix inequalities that are not solutions of the algebraic Riccati equation. The history of this result is typical of many results involving Riccati equations in the engineering literature-total confusion. It has been part of the folklore for many years that the solutions of the algebraic Riccati equation are extreme points of the above-mentioned quadratic matrix inequalities. In Badawi's thesis [1] a very elegant proof is given, however, in review it was discovered that there had in fact appeared a proof in the literature. In Faurre et al. [3] there is indeed a proof and a footnote to the effect that there are extreme points other than the solutions of the algebraic Riccati equation. However, it has evolved as part of the folklore that the two sets coincide, even though it seems to be known that there are extreme points that are not solutions of the algebraic Riccati equation. However, we have been unable to find a proof. In this note we present a class of examples that establishes that there are other extreme points.

The example is based on the simple analysis presented in [2]. Following the notation of [1] we let $F, G$ and $H$ and $R$ be matrices such that $F$ is $2 \times 2, G$ is $2 \times 1$, and $H$ is $1 \times 2$. We define then the function $W(P)=F P$ $+P F^{\prime}+\left(G-P H^{\prime}\right) R^{-1}\left(G-P H^{\prime}\right)^{\prime} . W(P)$ is the Riccati operator. The matrix Riccati inequality referred to above is of course the inequality $W(P) \leqslant 0$ in the sense of positive definite matrices. The Riccati equation is the equation $W(P)=0$. Now we choose $F, G$, and $H$ such that the Hamiltonian associated with the Riccati equation has complex eigenvalues. The Hamiltonian is constructed by transforming the above equation to the more standard form (for the purposes of geometric analysis)

$$
W(P)=\left(F-G R^{-1} H\right) P+P\left(F-G R^{-1} H\right)^{\prime}+G R^{-1} G^{\prime}+P H^{\prime} R^{-1} H P
$$

and writing the Hamiltonian $\mathscr{H}$

$$
\mathscr{H}=\left(\begin{array}{rr}
A & D \\
-Q & -A^{\prime}
\end{array}\right)
$$

where we let $A=F-G R^{-1} H, D=-H^{\prime} R^{-1} H$, and $Q=G R^{-1} G^{\prime}$. This matrix has four complex eigenvalues which can be denoted by $r, r,-r,-r$. (A standard result about infinitesimal symplectic matrices.) It is trivial to establish that such matrices exist. For example, let $A$ have complex eigenvalues and let $Q=s I$. Since $\mathscr{H}$ has complex eigenvalues with $s=0$, it follows (from continuity) that when $s$ is sufficiently small, the eigenvalues of the Hamiltonian are also. Choose the matrices $A, B, Q$. and $D$ such that $(A, H)$ is controllable and $Q$ and $D$ are positive semidefinite. Then using the results of [2] there exist exactly two real solutions of the associated algebraic Riccati equation. Thus, if the set of extreme points of the quadratic matrix inequalities consists only of these two solutions then the solution set is a linear segment. This is not the case and hence there must exist other extreme points.

Manuscript received January 9, 1984. This work was supported in part by the National Science Foundation under Grant ECS-7903731, in part by the Air Force Office of Scientific Research under Grant AFOSR78-3519, and in part by NASA under Grant 2384 and DOE Contract DE-AC01-80RA-5256.

A. Lindquist is with the Department of Mathematics. University of Kentucky, Lexington. KY 40506 and the Royal Institute of Technology. Stockholm, Sweden.

C. Martin is with the Department of Mathematics. Case Western Reserve University Cleveland. $\mathrm{OH} 44106$.

G. Picci is with LADSEB-CNR, Padova, taly

\section{REFERENCES}

[1] F. A. Badawi, "Structures and algorithms in stochastic realization theory and the smoothing problem," Ph.D. dissertation, Univ. Kentucky, 1980

[2] C. Martin, "Grassmannian manifolds, Riccati equation and feedback invariants of linear systems," in Geometrical Methods for the Theory of Linear Systems, C. Brynes and C. Martin, Eds. Dordrecht, The Netherlands: Riedel, 1981

[3] P. Faurre, M. Glerget. and F. Germain, Operaturs Rationel Postifs. Dunod, 1979.

\section{The Optimal Projection Equations for Fixed-Order Dynamic Compensation}

\section{DAVID C. HYLAND AND DENNIS S. BERNSTEIN}

Abstract-First-order necessary conditions for quadratically optimal, steady-state, fixed-order dynamic compensation of a linear, time-invariant plant in the presence of disturbance and observation noise are derived in a new and highly simplified form. In contrast to the pair of matrix Riccati equations for the full-order LQG case, the optimal steady-state fixed-order dynamic compensator is characterized by four matrix equations (two modified Riccati equations and two modified Lyapunov equations) coupled by a projection whose rank is precisely equal to the order of the compensator and which determines the optimal compensator gains. The coupling represents a graphic portrayal of the demise of the classical separation principle for the reduced-order controller case.

\section{INTRODUCTION}

Because of constraints imposed by on-line computations, dynamic controllers for high-order systems such as flexible spacecraft must be of relatively modest order. Hence, this paper is concerned with the design of quadratically optimal, fixed-order (i.e., reduced-order) dynamic compensation for a plant subject to stochastic disturbances and nonsingular measurement noise. Since white noise in all measurement channels precludes direct output feedback (see Section II), only purely dynamic controllers are considered. The requirements for resolution of this optimization problem include the following.

1) Conditions for the existence of an optimal, stabilizing compensator of the prescribed order. (In the full-order case these are the usual stabilizability and detectability conditions of LQG theory.)

2) Stationary conditions, i.e., first-order necessary conditions, rendered in a tractable form to facilitate developments in items 3) and 4) below. (In the full-order case these conditions are precisely the LQG gain relations together with the regulator and observer Riccati equations.)

3) Sufficiency conditions, i.e., additional restrictions on solutions of the first-order necessary conditions which characterize local minima and single out the global minimum. (In the full-order case the global minimum is distinguished by the unique nonnegative-definite solutions to the LQG Riccati equations.)

4) Convergent numerical algorithms for simultaneous satisfaction of the necessary and sufficient conditions. (In the full-order case numerical algorithms have been devised which take full advantage of the highly structured form of the Riccati equations.)

Manuscript received August 15, 1983; revised February 14, 1983. This paper is based on a prior submission of Marcb 10, 1983. Paper recommended by P. R. Kumar, Past Chairman of the Stochastic Control Committee. This work was supported by the Department of the Air Force and was performed at Lincoln Laboratory, M.I.T.

The authors are with the Controls Analysis and Synthesis Group, Harris Corp., GASD, Melbourne, FL 32902. 
The present paper deals exclusively with item 2). Although the stationary conditions for the fixed-order compensation problem have been written down (see [1]-[12], for example), full exploitation has undoubtedly been impeded by their extreme complexity [see (3.3)-(3.11)]. What has been lacking, to quote the insightful remarks of [9], "is a deeper understanding of the structural coherence of these equations." The contribution of the present paper is to show how the originally very complex stationary conditions can be transformed without loss of generality to much simpler and more tractable forms. The resulting equations (2.10)-(2.17) preserve the simple form of LQG relations for the gains in terms of covariance and cost matrices which, in turn, are determined by a coupled system of two modified Riccati equations and two modified Lyapunov equations. This coupling, by means of a projection (idempotent matrix) whose rank is pecisely equal to the order of the compensator, represents a graphic portrayal of the demise of the classical separation principle for the reduced-order controller case. When, as a special case, the order of the compensator is required to be equal to the order of the plant, the modified Riccati equations reduce to the standard LQG Riccati equations and the modified Lyapunov equations express the proviso that the compensator be minimal, i.e., controllable and observable. Since the LQG Riccati equations as such are nothing more than the necessary conditions for full-order compensation, we believe that the "optimal projection equations" provide a clear and simple generalization of standard LQG theory.

Since we are concerned with optimal fixed-order compensator design, our approach does not represent yet another model- or controllerreduction scheme along the lines of [13]-[17]. Indeed, the optimal projection equations, by virtue of their relatively transparent structure, can reveal the extent to which the design equations of a given ad hoc reduction scheme conform to the necessary conditions for optimality. For example, the oblique projection which arises in the present formulation may not be of the form $\left[\begin{array}{cc}I & 0 \\ 0\end{array}\right]$ even in the basis corresponding to the "balanced" realization [13]-[16]. These issues are discussed in [18] where the results of [19] are simplified by means of the approach of the present paper and where the balancing method of [13] is reinterpreted in the context of optimality theory.

The fact that the optimal projection equations consist of four coupled matrix equations, i.e., two modified Riccati equations and two modified Lyapunov equations, should not be at all surprising for the following simple reason. Reduced-order control-design methods often involve either LQG applied to a reduced-order model or model reduction applied to a full-order LQG design. Both approaches, then, involve the solution of precisely four equations: two Riccati equations (for LQG) plus two Lyapunov equations (for model reduction via balancing, as in [13]). The coupled form of the optimal projection equations is thus a strong reminder that the LQG and order-reduction operations cannot be iterated but must, in a certain sense, be performed simultaneously.

\section{PROblem Statement and the Main TheOREM}

Given the control system

$$
\begin{gathered}
\dot{x}(t)=A x(t)+B u(t)+w_{1}(t), \\
y(t)=C x(t)+w_{2}(t)
\end{gathered}
$$

design a fixed-order dynamic compensator

$$
\begin{gathered}
\dot{x}_{c}(t)=A_{c} x_{c}(t)+B_{c} y(t), \\
u(t)=C_{c} x_{c}(t)
\end{gathered}
$$

which minimizes the steady-state performance criterion

$$
J\left(A_{c}, B_{c}, C_{c}\right) \triangleq \lim _{t \rightarrow \infty}\left[{ }^{5}\left[x(t)^{T} R_{1} x(t)+u(t)^{T} R_{2} u(t)\right]\right.
$$

where: $x \in \mathbb{R}^{n}, u \in \mathbb{R}^{m}, y \in \mathbb{R}^{l}, x_{c} \in \mathbb{R}^{n} c, n_{c} \leqslant n, A, B, C, A_{c}, B_{c}$, $C_{c}, R_{1}$, and $R_{2}$ are matrices of appropriate dimension with $R_{1}$ (symmetric) nonnegative definite and $R_{2}$ (symmetric) positive definite; $w_{1}$ is white disturbance noise with $n \times n$ nonnegative-definite intensity $V_{1}$ and $w_{2}$ is white observation noise with $l \times l$ positive-definite intensity $V_{2} ; w_{1}$ and $w_{2}$ are uncorrelated and have zero mean. We note that the assumptions of nonsingular control weighting and nonsingular observation noise preclude the use of direct output feedback as in

$$
u(t)=C_{c} x_{c}(t)+D_{c} y(t)
$$

since $J$ is undefined unless (see [7])

$$
\operatorname{tr}\left[D_{c}^{T} R_{2} D_{c} V_{2}\right]=0 \quad\left(\Leftrightarrow R_{2} D_{c} V_{2}=0\right) .
$$

To guarantee that $J$ is finite and independent of initial conditions we restrict our attention to the set of admissible stabilizing compensators

$$
Q \triangleq\left\{\left(A_{c}, B_{c}, C_{c}\right): \tilde{A} \triangleq\left[\begin{array}{cc}
A & B C_{c} \\
B_{c} C & A_{c}
\end{array}\right] \text { is asymptotically stable }\right\}
$$

where $\tilde{A}$ is the closed-loop dynamics matrix. Since the value of $J$ is independent of the internal realization of the compensator, we can further restrict our attention to

$$
Q_{+} \triangleq\left\{\left(A_{c}, B_{\mathfrak{c}}, C_{c}\right) \in Q:\right.
$$

$$
\left.\left(A_{c}, B_{c}\right) \text { is controllable and }\left(C_{c}, A_{c}\right) \text { is observable }\right\} .
$$

For the following lemma call a square matrix nonnegative (respectively, positive) semisimple if it has a diagonal Jordan form and nonnegative (respectively, positive) eigenvalues. Let $I_{r}$ denote the $r \times r$ identity matrix.

Lemma 2.l: Suppose $\hat{Q}, \hat{P} \in \mathbb{R}^{n \times n}$ are nonnegative definite. Then $\hat{Q} \hat{P}$ is nonnegative semisimple. Furthermore, if rank $\hat{Q} \hat{P}=n_{c}$ then there exist $G, \Gamma \in \mathbb{R}^{n_{c} \times n}$ and positive-semisimple $M \in \mathbb{R}^{n} c^{\times n^{n}} c$ such that

$$
\begin{gathered}
\hat{Q P} \hat{P}=G^{T} M \Gamma, \\
\Gamma G^{T}=I_{n_{c}} .
\end{gathered}
$$

Proof. The result is an immediate consequence of $[20$, Theorem 6.2.5, p. 123].

For convenience in stating the Main Theorem, define

$$
\Sigma \triangleq B R_{2}^{-1} B^{T}, \bar{\Sigma} \triangleq C^{T} V_{2}^{-1} C
$$

and call $G, M$, and $\Gamma$ satisfying (2.8) and (2.9) a $(G, M, \Gamma)$-factorization of $\hat{Q} \hat{P}$.

Main Theorem: Suppose $\left(A_{c}, B_{c}, C_{c}\right) \in Q_{+}$solves the steady-state fixed-order dynamic-compensation problem. Then there exist $n \times n$ nonnegative-definite matrices $Q, P, \hat{Q}$, and $\hat{P}$ such that $A_{c}, B_{c}$, and $C_{c}$ are given by

$$
\begin{aligned}
& A_{c}=\Gamma(A-Q \bar{\Sigma}-\Sigma P) G^{T}, \\
& B_{c}=\Gamma Q C^{T} V_{2}^{-1}, \\
& C_{c}=-R_{2}^{-1} B^{T} P G^{T}
\end{aligned}
$$

for some $(G, M, \Gamma)$-factorization of $\hat{Q} \hat{P}$, and such that with $\tau \triangleq G^{T} \Gamma$ the following conditions are satisfied:

$$
\begin{gathered}
0=(A-\tau Q \bar{\Sigma}) Q+Q(A-\tau Q \bar{\Sigma})^{T}+V_{1}+\tau Q \bar{\Sigma} Q \tau^{T}, \\
0=(A-\Sigma P \tau)^{T} P+P(A-\Sigma P \tau)+R_{1}+\tau^{T} P \Sigma P \tau, \\
0=\tau\left[(A-\Sigma P) \hat{Q}+\hat{Q}(A-\Sigma P)^{T}+Q \bar{\Sigma} Q\right], \\
0=\left[(A-Q \bar{\Sigma})^{T} \hat{P}+\hat{P}(A-Q \bar{\Sigma})+P \Sigma P\right] \tau, \\
\text { rank } \hat{Q}=\operatorname{rank} \hat{P}=\operatorname{rank} \hat{Q} \hat{P}=n_{c} .
\end{gathered}
$$

Remark 2.1: Because of (2.9) the $n \times n$ matrix $\tau$ which couples the four equations (2.13)-(2.16) is idempotent, i.e., $\tau^{2}=\tau$. In general this "optimal projection" is an oblique projection (as opposed to an orthogonal projection) since it is not necessarily symmetric. Note that Sylvester's inequality and (2.9) imply that rank $\tau=n_{c}$.

Remark 2.2: Using the relations $\hat{Q}=\tau \hat{Q}$ and $\hat{P}=\hat{P} \tau[\sec (3.12)]$, 
the optimal projection equations $(2.13)-(2.16)$ can be written in the equivalent form

$$
\begin{gathered}
0=A Q+Q A^{T}+V_{1}-Q \bar{\Sigma} Q+\tau_{\perp} Q \bar{\Sigma} Q \tau_{\perp}^{T}, \\
0=A^{T} P+P A+R_{1}-P \Sigma P+\tau_{\perp}^{T} P \Sigma P \tau_{\perp}, \\
0=(A-\Sigma P) \hat{Q}+\hat{Q}(A-\Sigma P)^{T}+Q \bar{\Sigma} Q-\tau_{\perp} Q \bar{\Sigma} Q \tau_{\perp}^{T}, \\
0=(A-Q \bar{\Sigma})^{T} \hat{P}+\hat{P}(A-Q \bar{\Sigma})+P \Sigma P-\tau_{\perp}^{T} P \Sigma P \tau_{\perp}
\end{gathered}
$$

where $\tau_{\perp} \triangleq I_{n}-\tau$. Note that in the full-order case $n_{c}=n, \tau=G=\Gamma$ $=I_{n}$ and thus (2.18) and (2.19) reduce to the standard observer and regulator Riccati equations and (2.10)-(2.12) yield the usual LQG expressions. Furthermore, it can be shown that (2.20), (2.21), and (2.17) are equivalent to the assumption that $\left(A_{c}, B_{c}, C_{c}\right)$ is controllable and observable.

Remark 2.3: Since $\hat{Q} \hat{P}$ is nonnegative semisimple it has a group generalized inverse $(\hat{Q} \hat{P})^{*}$ given by $G^{T} M^{-1} \Gamma$ (see e.g., [21, p. 124]). Hence, by (2.9) the optimal projection $\tau$ is given by

$$
\tau=\hat{Q} \hat{P}(\hat{Q} \hat{P})^{\sharp} .
$$

Remark 2.4: The modified Riccati equations (2.13) and (2.14) are similar to the (single) "extended algebraic Riccati equation" which arises in the static output feedback problem (see, e.g., [22]).

Remark 2.5: Replacing $x_{c}$ by $S x_{c}$, where $S$ is invertible, yields the "equivalent" compensator $\left(S A_{c} S^{-1}, S B_{c}, C_{c} S^{-1}\right)$. Since $J\left(A_{c}, B_{c}, C_{c}\right)$ $=J\left(S A_{c} S^{-1}, S B_{c}, C_{c} S^{-1}\right)$ one would expect the Main Theorem to apply also to $\left(S A_{c} S^{-1}, S B_{c}, C_{c} S^{-1}\right)$. This is indeed the case since transformation of the compensator state basis corresponds to the alternative factorization $\hat{Q} \hat{P}=\left(S^{-T} G\right)^{T}\left(S M S^{-1}\right)(S T)$. See [10] for related remarks.

Remark 2.6: By introducing the quasi-full-state estimate $\hat{x} \triangleq G^{T} x_{c} \in$ $\mathbb{R}^{n}$ so that $\tau \hat{x}=\hat{x}$ and $x_{c}=\Gamma \hat{x} \in R^{n} c,(2.1)-(2.4)$ can be written as

$$
\begin{gathered}
\dot{x}=A x+B \hat{C}_{c} \tau \hat{x}+w_{1}, \\
\hat{x}=\tau\left(A-\hat{B}_{c} C+B \hat{C}_{c}\right) \tau \hat{x}+\tau \hat{B}_{c}\left(C x+w_{2}\right)
\end{gathered}
$$

where $\hat{B}_{c} \triangleq Q C^{T} V_{2}^{-1}$ and $\hat{C}_{c} \triangleq-R_{2}^{-1} B^{T} P$. Although the implemented compensator has the state $x_{c} \in R^{n} c$, it can be viewed as a quasi-full-order compensator whose geometric structure is entirely dictated by the projection $\tau$. Sensor inputs $\hat{B}_{c} y$ are annihilated unless they are contained in $[\mathscr{R}(\tau)]^{\perp}=\mathscr{R}\left(\tau^{T}\right)$, where $\mathfrak{N}$ and $R$ denote null space and range. Furthermore, the quasi-full-order state estimate $\tau \hat{x}$ employed in the control input is contained in $R(\tau)$. Thus, $\mathcal{R}(\tau)$ and $R\left(\tau^{T}\right)$ are the control and observation subspaces of the compensator.

\section{PROOF OF THE MAIN THEOREM}

The proof given here considerably simplifies the original derivation given in [23] and [24]. Using the fact that $a_{+}$is open, the Fritz John version of the Lagrange multiplier theorem can be used to rigorously derive the first-order necessary conditions ([7], see also [25])

$$
\begin{aligned}
& 0=\tilde{A} \tilde{Q}+\tilde{Q} \tilde{A}^{r}+\tilde{V}, \\
& 0=\tilde{A}^{T} \tilde{P}+\tilde{P} \tilde{A}+\tilde{R}, \\
& 0=P_{12}^{T} Q_{12}+P_{2} Q_{2}, \\
& B_{\mathrm{c}}=-\left(P_{2}^{-1} P_{12}^{T} Q_{\mathrm{l}}+Q_{12}^{T}\right) C^{T} V_{2}^{-1}, \\
& C_{c}=-R_{2}^{-1} B^{T}\left(P_{1} Q_{12} Q_{2}^{-1}+P_{12}\right),
\end{aligned}
$$

where

$$
\tilde{V} \triangleq\left[\begin{array}{cc}
V_{1} & 0 \\
0 & B_{c} V_{2} B_{c}^{T}
\end{array}\right], \quad \tilde{R} \triangleq\left[\begin{array}{cc}
R_{1} & 0 \\
0 & C_{c}^{T} R_{2} C_{c}
\end{array}\right]
$$

and $\left(n+n_{c}\right) \times\left(n+n_{c}\right) \tilde{Q}, \tilde{P}$ are partitioned into $n \times n, n \times n_{c}$, and $n_{c} \times n_{c}$ subblocks as

$$
\tilde{Q}=\left[\begin{array}{ll}
Q_{1} & Q_{12} \\
Q_{12}^{T} & Q_{2}
\end{array}\right], \quad \tilde{P}=\left[\begin{array}{cc}
P_{1} & P_{12} \\
P_{12}^{T} & P_{2}
\end{array}\right]
$$

Expanding (3.1) and (3.2) yields

$$
\begin{aligned}
& 0=A Q_{1}+Q_{1} A^{T}+B C_{c} Q_{12}^{T}+Q_{12}\left(B C_{c}\right)^{T}+V_{1}, \\
& 0=A Q_{12}+Q_{12} A_{c}^{T}+B C_{c} Q_{2}+Q_{1}\left(B_{c} C\right)^{T}, \\
& 0=A_{c} Q_{2}+Q_{2} A_{c}^{T}+B_{c} C Q_{12}+Q_{12}^{T}\left(B_{c} C\right)^{T}+B_{c} V_{2} B_{c}^{T}, \\
& 0=A^{T} P_{1}+P_{1} A+\left(B_{c} C\right)^{T} P_{12}^{T}+P_{12} B_{c} C+R_{1}, \\
& 0=P_{12} A_{c}+A^{T} P_{12}+\left(B_{c} C\right)^{T} P_{2}+P_{1} B C_{c}, \\
& 0=A_{c}^{T} P_{2}+P_{2} A_{c}+\left(B C_{c}\right)^{T} P_{12}+P_{12}^{T} B C_{c}+C_{c}^{T} R_{2} C_{c} .
\end{aligned}
$$

Writing (3.8) as (see [26], [27])

$$
0=\left(A_{c}+B_{c} C Q_{12} Q_{2}^{+}\right) Q_{2}+Q_{2}\left(A_{c}+B_{c} C Q_{12} Q_{2}^{+}\right)^{T}+B_{c} V_{2} B_{c}^{T}
$$

where $Q_{2}^{+}$is the Moore-Penrose or Drazin generalized inverse of $Q_{2}$, it follows from [28, Lemmas 2.1 and 12.2] that $Q_{2}$ is positive definite. Similarly, (3.11) implies that $P_{2}$ is positive definite. This justifies (3.4) and (3.5).

Now define the $n \times n$ nonnegative-definite matrices (see [26], [27])

$$
\begin{array}{ll}
Q \triangleq Q_{1}-Q_{12} Q_{2}^{-1} Q_{12}^{T}, & P \triangleq P_{1}-P_{12} P_{2}^{-1} P_{12}^{T}, \\
Q \triangleq Q_{12} Q_{2}^{-1} Q_{12}^{T}, & \hat{P} \triangleq P_{12} P_{2}^{-1} P_{12}^{T}
\end{array}
$$

and note that (3.3) implies (2.8) and (2.9) with

$$
G \triangleq Q_{2}^{-1} Q_{12}^{T}, M \triangleq Q_{2} P_{2}, \Gamma \triangleq-P_{2}^{-1} P_{12}^{T} .
$$

Since $Q_{2} P_{2}=P_{2}^{-1 / 2}\left(P_{2}^{1 / 2} Q_{2} P_{2}^{1 / 2}\right) P_{2}^{1 / 2}, M$ is positive semisimple. Sylvester's inequality yields (2.17). Note also that

$$
\hat{Q}=\tau \hat{Q}, \hat{P}=\hat{P} \tau \text {. }
$$

Next (2.11) and (2.12) follow from (3.4) and (3.5) by using the identities

$$
\begin{aligned}
& Q_{1}=Q+\hat{Q}, P_{1}=P+\hat{P}, \\
& Q_{12}=\hat{Q} \Gamma^{T}, P_{12}=-\hat{P} G^{T}, \\
& Q_{2}=\Gamma \hat{Q} \Gamma^{T}, P_{2}=G \hat{P} G^{T} .
\end{aligned}
$$

Now substitute (2.11), (2.12), and (3.13)-(3.15) into (3.6)-(3.11) and use the relations

$$
\begin{gathered}
B_{c} C=\Gamma Q \bar{\Sigma}, B C_{c}=-\Sigma P G^{T}, \\
B_{c} V_{2} B_{c}^{T}=\Gamma Q \bar{\Sigma} Q \Gamma^{T}, C_{c}^{T} R_{2} C_{c}=G P \Sigma P G^{T} .
\end{gathered}
$$

Then (2.10) follows from (3.8)- $\Gamma$ (3.7). Substituting (2.10) into (3.7), (3.8), (3.10), and (3.11) shows that $((3.7) G)^{T}$ and $-(3.10) \Gamma$ are precisely (2.15) and (2.16). Since $G^{T}(3.8) G=(2.15) \tau$ and $\Gamma^{T}(3.11) \Gamma=$ $\tau(2.16),(3.8)$ and $(3.11)$ can be omitted. Finally, using (3.12) it follows that $(2.13)=(3.6)+(2.15) \tau-(2.15)-(2.15)^{T}$ and similarly for (2.14).

\section{DIRECTIONS FOR FURTHER RESEARCH}

With regard to the existence of a stabilizing compensator, known results (e.g., [28]-[34]) can be exploited to a great extent. A numerical algorithm for solving the optimal projection equations has been developed in [24] and [35]. The proposed computational scheme is philosophically quite different from gradient search algorithms [2], [3], [6], [7], [9], [11], [36], [37] in that it operates through direct solution of the optimal projection equations by iterative refinement of the optimal projection. Methods for eliminating local extrema are being investigated by applying component cost analysis [17]. Generalizations of the optimal projection equations can arise by considering the following extensions of the fixedorder dynamic-compensation problem.

1) Discrete-Time System/Discrete-Time Compensator: Digital implementation can be modeled by a discrete-time compensator with control of a continuous-time system facilitated by sampling and reconstruction devices. 
2) Cross Weighting/Correlated Disturbance and Observation Noise: This extension is straighforward and entirely analogous to the LQG case (see, e.g., [3, p. 351]).

3) Singular Observation Noise/Singular Control Weighting: With due attention to (2.7), direct output feedback can be used in the singular case. The nature of the problem forebodes all of the difficulties associated with the singular LQG problem. Note that the output feedback problem [22], [38], when viewed in this context, is highly singular.

4) Infinite-Dimensional Systems: The optimal projection equations have been extended in [39] and [40] to the case in which (2.1) is a distributed parameter system, for example, a partial or functional differential equation.

5) Decentralized Fixed-Order Controller: The optimal projection equations can be derived for the case in which the dynamic controller has a fixed decentralized structure.

6) Parameter Uncertainties: The original derivation in [23] treated a Stratonovich state-dependent noise model representing parameter uncertainties in the plant. Further consideration of control- and measurementdependent noise raises the possibility of directly including the impact of parameter uncertainties in the design of robust, implementable compensation for large-order systems.

\section{REFERENCES}

[1] T. L. Johnson and M. Athans, "On the design of optimal constrained dynamic compensators for linear constant systems," IEEE Trans. Automat. Contr., vol. AC-15, pp. 658-660, 1970.

[2] W. S. Levine, T. L. Johnson, and M. Athans, "Optimal limited state variable feedback controllers for linear systems," IEEE Trans. Automat. Contr., vol. AC-16, pp. 785-793, 1971.

[3] K. Kwakemaak and R. Sivan, Linear Optimal Control Systems. New York: Wiley, 1972.

[4] D. B. Rom and P E. Sarachik, "The design of optimal compensators for linear constant systems wth inaccessible states," IEEE Trans. Automat. Contr,, vol. AC-18, pp. 509-512, 1973

[5] M. Sidar and B.-Z. Kurtaran, "Optimal low-order controllers for linear stochastic systems," Int. J. Contr., vol. 22, pp. 377-387, 1975.

[6] J. M. Mendel and J. Feather, "On the design of optimal time-invariant compensators for linear stochastic time-invariant systems," IEEE Trans. Automat. Contr., vol. AC-20, pp. 653-657, 1975.

[7] S. Basuthakur and C. H. Knapp, "Optimal constant controllers for stochastic linear systems," IEEE Trans. Automat. Contr., vol. AC-20, pp. 664-666, 1975.

[8] R. B. Asher and J. C. Durrett, "Linear discrete stochastic control with a reducedorder dynamic compensator," IEEE Trans. Automat. Contr., vol. AC-21, pp. 626-627, 1976.

[9] W. J. Naeije and O. H. Bosgra, "The design of dynamic compensators for linear multivariable systems," in Proc. 1977 IFAC, Fredricton, N. B., Canada, 1977, pp. $205-212$.

[10] P. J. Blanvillain and T. L. Johnson, "Invariants of optimal minimal-order observer based compensators," IEEE Trans. Automat. Contr., vol. AC-23, pp. $473-474,1978$.

[11] C. J. Wenk and C. H. Knapp, "Parameter optimization in linear systems with arbitrarily constrained controller structure," IEEE Trans. Automat. Contr., vol. AC-25, pp. 496-500, 1980

[12] J. O'Reilly, "Optimal low-order feedback controllers for linear discrete-time systems," in Control and Dynamic Systems, Vol. 16, C. T. Leondes, Ed. New York: Academic, 1980

[13] B. D. Moore, "Principal component analysis in linear systems: Controllability, observability and model reduction," IEEE Trans. Automat. Contr., vol. AC26, pp. 17-32, 1981.

[14] E. I. Verriest, "Suboptimal LQG-design and balanced realizations," in Proc. 20th IEEE Conf. Decision Contr., San Diego, CA, Dec. 1981, pp. 686-687.

[15] L. Pernebo and L. M. Silverman, "Model reduction via balanced state space representations," IEEE Trans. Automat. Contr., vol. AC-27, pp. 382-387, 1982.

[16] E. A. Jonckheere and L. M. Silverman, "A new set of invariants for linear systems-application to reduced-order compensator design," IEEE Trans. Automat. Contr., vol. AC-28, pp. 953-964, 1983.

[17] A. Yousuff and R. E. Skelton, "Controller reduction by component cost analysis," IEEE Trans. Automat. Contr., vol. AC-29, pp. 520-530, 1984

[18] D. C. Hyland and D. S. Bernstein, "The optimal projection approach to model reduction and the relationship between the methods of Wilson and Moore," in 23rd IEEE Conf. Decision Contr.. Las Vegas. NV. Dec. 1984

[19] D. A. Wilson, "Optimum solution of model-reduction problem," Proc. IEE, vol. 117, pp. 1161-1165, 1970.

[20] C. R. Rao and S. K. Mitra, Generalized Inverse of Matrices and its Applications. New York: Wiley, 1971.
[21] S. L. Campbell and C. D. Meyer, Jr., Generalized Inverses of Linear Transformations, London: Pitman, 1979.

[22] J. Medanic, "On stabilization and optimization by output feedback," in Proc. 12th Annual Asilomar Conf. Circuits and Syst., 1978, pp. 412-416.

[23] D. C. Hyland, "Optimality conditions for fixed-order dynamic compensation of flexible spacecraft with uncertain parameters," in Proc. AIAA 20th Aerospace Sciences Meet., Orlando, FL, Jan. 1982, paper 82-0312.

[24] D. C. Hyland, "The optimal projection approach to fixed-order compensation: Numerical methods and illustrative result,"' in AIAA 21st Aerospace Sciences Meet., Reno, NV, Jan. 1983, paper 83-0303.

[25] D. C. Hyland and D. S. Bernstein, "Explicit optimality conditions for fixed-order dynamic compensation," in Proc. 22nd IEEE Conf. Decision Contr., San Antonio, TX, Dec. 1983.

[26] A. Albert, "Conditions for positive and nonnegative definiteness in terms of pseudo inverse," SIAM J. Appl. Math., vol. 17, pp. 434-440, 1969.

[27] E. Kreindler and A. Jameson, "Conditions for nonnegativeness of partitioned matrices," IEEE Trans. Automat. Contr., vol. AC-17, pp. 147-148, 1972.

[28] W. M. Wonham, Linear Multivariable Control: A Geometric Approach. New York: Springer-Verlag, 1974.

[29] F. M. Brasch and J. B. Pearson, "Pole placement using dynamic compensators," IEEE Trans. Automat. Contr., vol. AC-15, pp. 34-43, 1970.

[30] R. Ahmari and A. G. Vacroux, "On the pole assignment in linear systems with fixed-order compensators," Int. J. Contr., vol. 17, pp. 397-404, 1973.

[31] D. C. Youla, J. J. Bongiorno, Jr., and C. N. Lu, "Single-loop feedback stabilization of linear multivariable dynamical plants," Automatica, vol. 10, pp. $159-173,1974$.

[32] H. Seraji, "An approach to dynamic compensator design for pole assignment," Int. J. Contr., vol. 21, pp. 955-966, 1975.

[33] R. V. Patel, "Design of dynamic compensators for pole assignment," Int. J. Syst. Sci., vol, 7, pp. 207-224, 1976.

[34] C. A. Desoer, R. W. Liu, J. Murrary, and R. Saeks, "Feedback system design: The fractional representation approach to analysis and synthesis,' IEEE Trans. Automat. Contr., vol. AC-25, pp. 399-412, 1980.

[35] D. C. Hyland, "Comparison of various controller-reduction methods: Suboptimal versus optimal projection," in Proc. AIAA Dynamics Specialists Conf., Palm Springs, CA, May 1984, pp. 381-389.

[36] H. R. Sirisena and S. S. Choi, "Design of optimal constrained dynamic compensators for non-stationary linear stochastic systems," Int. J. Contr., vol. 25, pp. 513-524, 1977.

[37] D. P. Looze and N. R. Sandell, Jr., "Gradient calculations for linear quadratic fixed control structure problems," IEEE Trans. Automat. Contr., vol. AC-25, pp. 285-288, 1980.

[38] W. S. Levine and $M$. Athans, "On the determination of the optimal constant output feedback gains for linear multivariable systems," IEEE Trans. Automat. Contr., vol. AC-15, pp. 44-48, 1970.

[39] D. S. Bernstein and D . C. Hyland, "The optimal projection equations for fixedorder dynamic compensation of distributed parameter systems," in Proc. ALAA Dynamics Specialisis Conf., Palm Springs, CA, May 1984, pp. 396-400.

[40] D. S. Bernstein, "Explicit optimality conditions for finite-dimensional fixed-order dynamic compensation of infinite-dimensional systems," submitted for publication.

\section{Optimal Control of Systems Possessing Symmetries}

\section{J. W. GRIZZLE AND S. I. MARCUS}

Abstract-It is shown that a symmetry in an optimization problem induces a decomposition of the optimal feedback control law into factors. One factor can be calculated algebraically and depends only on the symmetry; the other factor corresponds to a lower dimensional optimization problem. This gives a priori information about the structure of the optimal feedback control law and indicates a possibly more efficient method for optimizing such systems.

Manuscript received November 21, 1983; revised May 21, 1984. This work was supported in part by the Air Force Office of Scientific Research under Grant AFOSR-790025, in part by the National Science Foundation under Grant ECS-8022033, and in part by the Joint Services Electronics Program under Contract F49620-82-C-0033.

J. W. Grizzlc was with the Department of Electrical Engineering, The University of Texas at Austin, Austin, TX 78712. He is now with the Department of Electrical and Computer Engineering, University of Illinois, Urbana, IL 61801.

S. I. Marcus is with the Department of Electrical and Comptuer Engineering, The University of Texas at Austin, Austin, TX 78712. 